\title{
Continuity of the Effective Delay Operator for Networks Based on the Link Delay Model
}

\author{
Ke Han' ${ }^{1}$. Terry L. Friesz ${ }^{2}$
}

Published online: 14 December 2017

(C) The Author(s) 2017. This article is an open access publication

\begin{abstract}
This paper is concerned with a dynamic traffic network performance model, known as dynamic network loading (DNL), that is frequently employed in the modeling and computation of analytical dynamic user equilibrium (DUE). As a key component of continuous-time DUE models, DNL aims at describing and predicting the spatial-temporal evolution of traffic flows on a network that is consistent with established route and departure time choices of travelers, by introducing appropriate dynamics to flow propagation, flow conservation, and travel delays. The DNL procedure gives rise to the path delay operator, which associates a vector of path flows (path departure rates) with the corresponding path travel costs. In this paper, we establish strong continuity of the path delay operator for networks whose arc flows are described by the link delay model (Friesz et al., Oper Res 41(1):80-91, 1993; Carey, Networks and Spatial Economics 1(3):349-375, 2001). Unlike the result established in Zhu and Marcotte (Transp Sci 34(4):402-414, 2000), our continuity proof is constructed without assuming a priori uniform boundedness of the path flows. Such a more general continuity result has a few important implications to the existence of simultaneous route-and-departure-time DUE without a priori boundedness of path flows, and to any numerical algorithm that allows convergence to be rigorously analyzed.
\end{abstract}

Ke Han

k.han@imperial.ac.uk

Terry L. Friesz

tfriesz@psu.edu

1 Department of Civil and Environmental Engineering, Imperial College London,

London SW7 2BU, UK

2 Department of Industrial and Manufacturing Engineering, Pennsylvania State University, University Park, State College, PA 16802, USA 
Keywords Dynamic traffic assignment - Dynamic user equilibrium - Dynamic network loading $\cdot$ Effective delay operator $\cdot$ Link delay model

\section{Introduction}

Dynamic traffic assignment (DTA) is usually viewed as the descriptive modeling of time varying flows on vehicular networks consistent with established traffic flows. It stems from the need to simultaneously model travelers' choices and vehicular flow characteristics in a dynamic and network context (Ben-Akiva et al. 2015; GoyaGómez et al. 2017). For a general review of a broad class of DTA models and problems, we refer the reader to Peeta and Ziliaskopoulos (2001), Szeto and Lo (2006) and Nesterov and De Palma (2003).

This paper is concerned with a specific type of DTA problem known as simultaneous route-and-departure-time (SRDT) dynamic user equilibrium (DUE) in continuous time. In this model, the unit travel cost experienced by road users of the same origin-destination pair, including path travel time and arrival penalty, is identical and minimum. There are two essential components within the notion of DUE: (i) the mathematical expression of Nash-like equilibrium conditions, and (ii) a network performance model, which is, in effect, an embedded dynamic network loading (DNL) problem.

The Nash-like equilibrium condition of a DUE problem is the dynamic extension of Wardrop's first principle (Wardrop 1952), which admits multiple mathematical representations. These include variational inequality (Friesz et al. 1993, 2013; Han et al. 2013c; Szeto and Lo 2004); differential variational inequality (Freisz et al. 2001, 2010; Friesz and Mookherjee 2006); nonlinear complementarity problem (Ukkusuri et al. 2012; Wie et al. 2002); and differential complementarity problem (Pang et al. 2011). In addition, several extensions of the DUE problem pertaining to bounded user rationality (Han et al. 2015b), demand elasticity (Friesz and Meimand 2014; Han et al. 2015a) and en-route update (Kachroo and Özbay 2005), have been formulated using (differential) variational inequalities (which are closely related to open-loop optimal control problems) and feedback (closed-loop) control formulations.

On the other hand, the network performance model, often referred to as the dynamic network loading (DNL) model, is concerned with describing and predicting the spatial-temporal evolution of traffic flows on a network, which is consistent with established route and departure time choices of drivers. This is done by introducing appropriate dynamics to link flow, junction flow, link delay, and path delay, which are usually constrained by the first-in-first-out (FIFO) rule (Zhang and Nie 2005).

The DNL model gives rise to the path delay operator or effective path delay operator, whose precise definitions will be explicitly given in this paper. The (effective) delay operators play a pivotal role in the mathematical formulations of DUE problem, including variational inequality (Friesz et al. 1993), differential variational inequality (Friesz et al. 2001; Friesz and Mookherjee 2006), and complementarity problems (Pang et al. 2011; Wie et al. 2002). Continuity of the effective delay operator, as we focus in this paper, is critical to the DUE models as it is necessary 
for the existence of dynamic user equilibria (Browder 1968; Han et al. 2013c; Zhu and Marcotte 2000). Moreover, the continuity of a delay operator is essential for any computational algorithms for DUE with a provable convergence result, such as fixed point algorithms (Friesz et al. 2010, 2013), descent direction methods (Han and Lo 2003; Szeto and Lo 2004), projection methods (Ukkusuri et al. 2012; Han and Lo 2002), and proximal point methods (Konnov 2003; Han et al. 2015a).

One way to model path delay in dynamic networks is that proposed by (Friesz et al. 1993), who employ link exit time functions together with a volume-dependent link traversal time function. Such a perspective on path delay has been referred to as the link-delay model (LDM). Since the Friesz et al. (1993) paper, conjectures but few rigorous results about the qualitative properties of the LDM delay operator have been published. One result that is needed for studying the existence of DUE and computing its solutions when the network loading is based on the LDM, is continuity of the effective path delay operator. It should be mentioned that Zhu and Marcotte (2000) investigate a similar problem and show weak continuity of the path delay operator under the assumption that the path flows are a priori bounded, where those bounds do not arise from any behavioral argument or theory. In particular, we note that Theorem 5.1 of Zhu and Marcotte (2000), which states the weak continuity of the delay operator, relies on the strong first-in-first-out (FIFO) assumption. Although the paper later stated that a sufficient condition for strong FIFO is uniform boundedness of path flows (path departure rates), it is not difficult to show that boundedness is also necessary to ensure strong FIFO. Effectively, the strong FIFO assumption is equivalent to the uniform boundedness of path flows.

Notably, this paper provides a more general continuity result, namely the path delay operator of interest is strongly continuous without the assumption of boundedness on path flows. Strong continuity without boundedness is central to the proof of SRDT DUE existence, and to the analyses of DUE algorithms. We point out that the simultaneous route-and-departure-time (SRDT) notion of DUE employs more general constraints relating path flows to a table of fixed trip volumes than the route choice (RC) DUE considered by Zhu and Marcotte (2000). The boundedness assumption is less of an issue for the RC DUE by virtue of problem formulation: that is, for RC DUE, the travel demand constraints are of the following form:

$$
\sum_{p \in \mathcal{P}_{i j}} h_{p}(t)=T_{i j}(t) \quad \forall t, \quad \forall(i, j) \in \mathcal{W}
$$

where $\mathcal{W}$ is the set of origin-destination pairs, $\mathcal{P}_{i j}$ is the set of paths connecting $(i, j) \in \mathcal{W}$ and $h_{p}(t)$ is the departure rate along path $p$. Furthermore, $T_{i j}(t)$ represents the rate (not volume) at which travelers leave origin $i$ with the intent of reaching destination $j$ at time $t$; each such trip rate is assumed to be bounded from above. Since Eq. (1) is imposed pointwise and every path flow $h_{p}$ is non-negative, we are assured that $h=\left(h_{p}: p \in \mathcal{P}_{i j},(i, j) \in \mathcal{W}\right)$ are automatically uniformly bounded. On the other hand, the SRDT user equilibrium imposes the following constraints on path flows:

$$
\sum_{p \in \mathcal{P}_{i j}} \int_{t_{0}}^{t_{f}} h_{p}(t) d t=Q_{i j} \quad \forall(i, j) \in \mathcal{W}
$$


where $Q_{i j} \in \mathbb{R}_{++}$is the volume (not rate) of travelers departing from node $i$ with the intent of reaching node $j$. The integrals in Eq. (2) are interpreted in the sense of Lebesgue; hence, Eq. (2) alone is not enough to assure bounded path flows. This observation has been the major hurdle to providing existence without the invocation of bounds on path flows, and therefore, serves as the main motivation of our investigation of the continuity of the delay operator without assuming a priori boundedness on path flows.

\section{Background and Preliminaries}

\subsection{Notation}

A summary of sets, variables and parameters used in this paper is shown in Table 1.

Throughout this paper, we assume that the path departure rate $h_{p}(\cdot) \in L_{+}^{2}\left[t_{0}, t_{f}\right]$ and, hence, $h(\cdot) \in\left(L_{+}^{2}\left[t_{0}, t_{f}\right]\right)^{|\mathcal{P}|}$, which is a fairly general regularity condition

Table 1 Notations

$\begin{array}{ll}\text { Sets } & \\ {\left[t_{0}, t_{f}\right]:} & \text { Time horizon in which the problem is conceived; } t_{f}>t_{0} \\ G(\mathcal{A}, \mathcal{V}): & \text { Traffic network represented as a graph with arc set } \mathcal{A} \text { and node set } \mathcal{V} \\ \mathcal{P}: & \text { Set of paths } \\ \mathcal{W}: & \text { Set of origin-destination }(\mathrm{OD}) \text { pairs } \\ \mathcal{P}_{i j}: & \text { Set of paths connecting the OD pair }(i, j) \in \mathcal{W} \\ Q_{i j}: & \text { Demand for OD pair }(i, j) \in \mathcal{W} \\ L^{2}\left[t_{0}, t_{f}\right]: & \text { The set of square-integrable functions defined on }\left[t_{0}, t_{f}\right] \\ L_{+}^{2}\left[t_{0}, t_{f}\right]: & \text { The subset of } L^{2}\left[t_{0}, t_{f}\right] \text { consisting of non-negative functions } \\ \left(L^{2}\left[t_{0}, t_{f}\right]\right)^{m}: & \text { The } m \text {-fold product of the } L^{2}\left[t_{0}, t_{f}\right] \text { space } \\ \left(L_{+}^{2}\left[t_{0}, t_{f}\right]\right)^{m}: & \text { The subset of }\left(L^{2}\left[t_{0}, t_{f}\right]\right)^{m} \text { consisting of vectors whose elements are } \\ & \text { non-negative functions } \\ \mathbb{R}: & \text { The set of real numbers } \\ \mathbb{R}_{+}: & \text {The set of non-negative real numbers } \\ \mathbb{R}_{++}: & \text {The set of positive real numbers }\end{array}$

Variables

$h_{p}(t): \quad$ Departure rate along path $p \in \mathcal{P}$ at time $t \in\left[t_{0}, t_{f}\right]$

$h(t): \quad$ The vector of departure rates over all $p \in \mathcal{P}$ at $t \in\left[t_{0}, t_{f}\right]$

$D_{p}(t, h): \quad$ Travel time (delay) along path $p$ for departure time $t$ given the departure rate vector $h$

$\Psi_{p}(t, h): \quad$ Effective delay occurred on path $p$ for departure time $t$ given the departure rate vector $h$

Parameters

$\Delta=\left\{\delta_{a p}\right\}:$

The arc-path incidence matrix, $a \in \mathcal{A}, p \in \mathcal{P}$

$v_{i j}$ :

The essential infimum of the effective delays over all $p$ and $t$ for OD $(i, j)$ 
(Friesz et al. 1993, 2010; Han et al. 2013c). Such an assumption also plays a central role in the theory of existence and computation of DUE established in the Hilbert space $\left(L^{2}\left[t_{0}, t_{f}\right]\right)^{|\mathcal{P}|}$ (Friesz et al. 2013; Han et al. 2013c).

\subsection{Dynamic User Equilibrium and the Effective Delay Operator}

This paper is concerned with a form of dynamic traffic assignment (DTA) known as simultaneous route-and-departure-time (SRDT) dynamic user equilibrium (DUE) for which unit travel cost, including early and late arrival penalties, is identical for those route and departure time choices selected by travelers between a given origin-destination pair. Such a model is originally presented in Friesz et al. (1993) and discussed subsequently by Friesz et al. (2001, 2013, 2010), and Friesz and Mookherjee (2006).

We begin by considering a planning horizon $\left[t_{0}, t_{f}\right]$. Let $\mathcal{P}$ be the set of paths employed by road users. The most crucial component of the DUE model is the path delay operator. We let

$$
D_{p}(t, h) \quad \forall p \in \mathcal{P}, t \in\left[t_{0}, t_{f}\right]
$$

be the time spent traversing path $p$ with departure time $t$, when the network congestion is determined by the vector of path departure rates $h \in\left(L_{+}^{2}\left[t_{0}, t_{f}\right]\right)^{|\mathcal{P}|}$. Equation (3) induces the following operator, which is referred to as the delay operator:

$$
D:\left(L_{+}^{2}\left[t_{0}, t_{f}\right]\right)^{|\mathcal{P}|} \rightarrow\left(L_{+}^{2}\left[t_{0}, t_{f}\right]\right)^{|\mathcal{P}|}, \quad h \mapsto\left(D_{p}(\cdot, h): p \in \mathcal{P}\right)
$$

As adequately covered in a number of studies (Friesz et al. 2013, 2010; Zhu and Marcotte 2000), the path delay operators usually do not take on any closed form; instead they can only be evaluated numerically through the dynamic network loading procedure (Friesz et al. 2013; Han et al. 2016; Lo and Szeto 2002; Nie and Zhang 2010; Szeto and Lo 2004; Ukkusuri et al. 2012).

In the SRDT DUE model, the drivers choose departure times, in addition to paths, in order to minimize their travel costs. To accommodate such a choice behavior, a notion of travel cost more general than travel time needs to be introduced, by adding arrival penalties to the path delays. Specifically, we consider the following general form of effective path delay:

$$
\Psi_{p}(t, h)=D_{p}(t, h)+\mathcal{F}\left[t+D_{p}(t, h)-T_{A}\right] \quad \forall p \in \mathcal{P}, t \in\left[t_{0}, t_{f}\right]
$$

where $T_{A}$ is the target arrival time. In our formulation the target time $T_{A}$ is allowed to depend on the user classes (or origin-destination pairs). As shown in Eq. (5), the effective path delay assesses an additional (asymmetric) cost for not arriving at the target arrival time.

We introduce the fixed trip matrix $\left\{Q_{i j}:(i, j) \in \mathcal{W}\right\}$, where each $Q_{i j} \in \mathbb{R}_{+}$is the fixed travel demand between origin-destination pair $(i, j) \in \mathcal{W}$. Note that $Q_{i j}$

\footnotetext{
${ }^{1}$ For notation convenience and without causing any confusion, we sometimes use $h$ instead of $h(\cdot)$ to denote the path departure rate vectors.
} 
represents traffic volume, not flow; this is a key distinction between SRDT DUE and RC DUE as illustrated in Eqs. (1)-(2). The set of feasible path flows is now defined as:

$$
\Lambda=\left\{h \geq 0: \sum_{p \in \mathcal{P}_{i j}} \int_{t_{0}}^{t_{f}} h_{p}(t) d t=Q_{i j} \quad \forall(i, j) \in \mathcal{W}\right\} \subseteq\left(L_{+}^{2}\left[t_{0}, t_{f}\right]\right)^{|\mathcal{P}|}
$$

Let us also define the essential infimum of the effective path delay

$$
v_{i j}^{p}(h) \doteq \operatorname{essinf}_{t \in\left[t_{0}, t_{f}\right]} \Psi_{p}(t, h) \quad \forall p \in \mathcal{P}_{i j}, \quad(i, j) \in \mathcal{W}
$$

Essential infimum of a function is similar to the minimum of that function, except it has been generalized in a measure-theoretic context to treat measurable functions. We further define the minimum travel cost between an OD pair to be:

$$
v_{i j}(h) \doteq \min _{p \in \mathcal{P}_{i j}} v_{i j}^{p}(h) \quad \forall(i, j) \in \mathcal{W}
$$

We note that both $v_{i j}^{p}$ and $v_{i j}$ depend on the path departure rate vector $h$.

The following definition of dynamic user equilibrium was first articulated by Friesz et al. (1993):

Definition i (Dynamic user equilibrium). A vector of departure rates (path flows) $h^{*} \in \Lambda$ is a dynamic user equilibrium if

$$
h_{p}^{*}(t)>0, p \in P_{i j} \Longrightarrow \Psi_{p}\left(t, h^{*}(t)\right)=v_{i j}
$$

We denote this equilibrium by $D U E\left(\Psi, \Lambda,\left[t_{0}, t_{f}\right]\right)$.

Using measure theoretic arguments, Friesz et al. (1993) establish that a dynamic user equilibrium is equivalent to the following variational inequality.

$$
\left.\begin{array}{c}
\text { find } h^{*} \in \Lambda \text { such that } \\
\sum_{p \in \mathcal{P}} \int_{t_{0}}^{t_{f}} \Psi_{p}\left(t, h^{*}\right)\left(h_{p}-h_{p}^{*}\right) d t \geq 0 \\
\forall h \in \Lambda
\end{array}\right\} V I\left(\Psi, \Lambda,\left[t_{0}, t_{f}\right]\right)
$$

\subsection{The Link Delay Model}

First introduced in Friesz et al. (1993), the link delay model (LDM) is one type of link performance model where the link traversal time is a function of the link occupancy. In particular, let $x(t)$ be the number of vehicles on the link of interest, $\alpha, \beta>0$ be link parameters, then link travel time (delay) experienced by drivers entering the link at time $t$ is

$$
\mathcal{D}[X(t)]=\alpha X(t)+\beta
$$


The affine link delay function is selected as it remains the only known functional form that guarantees first-in-first-out (FIFO) (Nie and Zhang 2005), which is typically required for the theoretical investigation of DUE problems.

We denote by $u(t)$ and $v(t)$ the entry and exit flows of the link, respectively. In this paper, both functions are only assumed to be integrable, and we define the cumulative entry and exit counts as:

$$
U(t) \doteq \int_{t_{0}}^{t} u(s) d s, \quad V(t) \doteq \int_{t_{0}}^{t} v(s) d s
$$

For DTA problems it is common to assume that the link (and network) is initially empty. Therefore, by definition, the occupancy is computed as:

$$
X(t)=U(t)-V(t) \quad \forall t \in\left[t_{0}, t_{f}\right]
$$

The flow propagation constraint for the link of interest, which obeys the FIFO principle, is expressed as:

$$
U(t)=V(t+\mathcal{D}[X(t)]) \quad \forall t \in\left[t_{0}, t_{f}\right]
$$

Equations (12)-(13), together with the integral form (11) (or its differential form), constitute the fundamental link dynamic for the LDM. The next theorem reports an important property of the LDM and will be constantly invoked later in this paper.

Theorem i (Friesz et al. 1993). For any linear arc delay function of the form $D(x)=$ $\alpha x+\beta, \alpha, \beta>0$, the resulting arc exit time function $\tau$ is continuous and strictly increasing and hence the inverse function $\tau^{-1}$ exists.

Proof See Theorem 1 of Friesz et al. (1993).

\subsection{Dynamic Network Loading Based on the LDM}

Typically, a traffic network is represented as a directed graph $G(\mathcal{V}, \mathcal{A})$ where $\mathcal{V}$ is the set of nodes and $\mathcal{A}$ is the set of arcs/links. A path that connects an OD pair $(i, j) \in \mathcal{W}$ is expressed as an ordered sequence that it traverses:

$$
p \in \mathcal{P}, \quad p=\left\{a_{1}, a_{2}, \ldots, a_{m(p)}\right\} \subset \mathcal{A}
$$

where $m(p)$ denotes the number of arcs in the path. The path flow or path departure rate, $h_{p}(\cdot)$, is defined as the rate at which traffic enter the first arc of $p \in \mathcal{P}$.

We define $u_{a}^{p}(\cdot)$ and $v_{a}^{p}(\cdot)$ to be the entry and exit flows of arc $a$, respectively, that are associated with path $p$. With similar meaning of notation, we let $X_{a}^{p}(\cdot), U_{a}^{p}(\cdot)$, and $V_{a}^{p}(\cdot)$ be the arc volume, cumulative arc entry count, and cumulative arc exit count, respectively. All these variables are functions of time. The following DAE system (14)-(18) for the DNL 
procedure can be readily derived based on the link dynamics introduced in Section 2.3

$$
\begin{array}{rr}
\frac{U_{a_{i}}^{p}(t)}{d t}=u_{a_{i}}^{p}(t), \quad \frac{V_{a_{i}}^{p}(t)}{d t}=v_{a_{i}}^{p}(t) & \forall p \in \mathcal{P}, i \in\{1, \ldots, m(p)\} \\
\frac{d X_{a_{i}}^{p}(t)}{d t}=u_{a_{i}}^{p}(t)-v_{a_{i}}^{p}(t) & \forall p \in \mathcal{P}, \quad i \in\{1, \ldots, m(p)\} \\
u_{a_{1}}^{p}(t) \equiv h_{p}(t) & \forall p \in \mathcal{P}, a_{1} \in p \\
U_{a_{i}}^{p}(t)=V_{a_{i}}^{p}\left(t+\mathcal{D}_{a_{i}}\left[X_{a_{i}}(t)\right]\right) & \forall p \in \mathcal{P}, \quad i \in\{1, \ldots, m(p)\} \\
X_{a}(t)=\sum_{p \in \mathcal{P}} \delta_{a p} X_{a}^{p}(t) & \forall a \in \mathcal{A}
\end{array}
$$

In order to calculate link and path delays, we introduce the arc exit time function

$$
\tau_{a}(t) \doteq t+\mathcal{D}_{a}\left[X_{a}(t)\right] \quad \forall a \in \mathcal{A}
$$

As a result of solving the DAE system above, one may easily compute the arc exit time function $\tau_{a}(\cdot)$. Then, given that cars leaving the preceding arc enter the following arc without delay, we may compute the path travel time (path delay) function as a series of function compositions:

$$
D_{p}(t, h)=\tau_{a_{1}} \circ \tau_{a_{2}} \circ \ldots \circ \tau_{a_{m(p)}}(t)=\tau_{a_{m(p)}}\left(\tau_{a_{m(p)-1}}\left(\ldots \tau_{a_{1}}(t)\right)\right)
$$

where $p=\left\{a_{1}, a_{2}, \ldots, a_{m(p)}\right\}$. Finally, the effective path delays can be computed according to (5).

\section{The Main Result}

The following is a statement of our main result:

Theorem ii Consider a general network $(\mathcal{A}, \mathcal{V})$, where the arc dynamics are governed by the link delay model, assume the link delay function for each a $\in \mathcal{A}$ is affine. That is

$$
\mathcal{D}_{a}\left[x_{a}(t)\right]=\alpha_{a} X_{a}(t)+\beta_{a}
$$

where $\alpha_{a} \in \mathbb{R}_{++}$and $\beta_{a} \in \mathbb{R}_{++}$. Then, the effective delay operator from $\Lambda$ into $\left(L^{2}\left(\left[t_{0}, t_{f}\right]\right)^{|\mathcal{P}|}: h \in \Lambda \longrightarrow \Psi(\cdot, h)\right.$ is a continuous map.

Remark iii In Zhu and Marcotte (2000), the authors show that the effective delay operator is weakly continuous when the LDM is employed, under the restrictive assumption that the path flows are a priori bounded from above. Such assumption is dropped in our result; we also assert strong, not weak continuity.

The above theorem tells us there are no jump or other kinds of discontinuities of the path delay operator. Such an analytical result is crucial for studying the existence of dynamic user equilibrium as well as analyzing DUE algorithms, when the network loading is based on the LDM. 


\subsection{Proof of the Main Result}

Now we present the proof of Theorem ii.

Proof We begin by showing that given a converging sequence $h^{(n)}$ in the space $\Lambda \subset$ $\left(L_{+}^{2}\left(\left[t_{0}, t_{f}\right]\right)\right)^{|\mathcal{P}|}$ such that

$$
\left\|h^{(n)}-h\right\|_{L^{2}} \longrightarrow 0 \quad n \longrightarrow \infty,
$$

the corresponding delay function $D_{p}\left(\cdot, h^{(n)}\right)$ converges uniformly to $D_{p}(\cdot, h)$ for all $p \in \mathcal{P}$. This will be proven in several steps.

Part 1. First, let us consider just one single arc, and hence omit the subscript $a$ for brevity. Assume a sequence of entering flows $\left\{u^{(n)}\right\}_{n \geq 1}$ converging to $u$ in the $L^{2}\left(\left[t_{0}, t_{f}\right]\right)$ space; that is

$$
\left\|u^{(n)}-u\right\|_{2} \doteq\left(\int_{t_{0}}^{t_{f}}\left(u^{(n)}(t)-u(t)\right)^{2} d t\right)^{1 / 2} \longrightarrow 0 \quad n \rightarrow \infty
$$

Define the cumulative entering vehicle counts

$$
\begin{aligned}
& U^{(n)}(t) \doteq \int_{t_{0}}^{t} u^{(n)}(s) d s \quad n \geq 1 \\
& U(t) \doteq \int_{t_{0}}^{t} u(s) d s
\end{aligned}
$$

Then we assert the uniform convergence $U^{(n)} \longrightarrow U$ on $\left[t_{0}, t_{f}\right]$. To see this, fix any $\varepsilon>0$; in view of (22), choose $N>0$ such that for all $n>N$

$$
\left\|u^{(n)}-u\right\|_{2}<\varepsilon
$$

According to the embedding of $L^{1}\left(\left[t_{0}, t_{f}\right]\right)$ into $L^{2}\left(\left[t_{0}, t_{f}\right]\right)$, we deduce for any $t \in\left[t_{0}, t_{f}\right]$ that

$$
\begin{aligned}
\left|U^{(n)}(t)-U(t)\right| & =\left|\int_{t_{0}}^{t} u^{(n)}(s) d s-\int_{t_{0}}^{t} u(s) d s\right| \\
& \leq\left\|u^{(n)}-u\right\|_{1} \leq\left(t_{0}-t_{f}\right)^{1 / 2}\left\|u^{(n)}-u\right\|_{2} \\
& <\left(t_{0}-t_{f}\right)^{1 / 2} \varepsilon
\end{aligned}
$$

The preceding shows the uniform convergence $U^{(n)} \longrightarrow U$ on $\left[t_{0}, t_{f}\right]$.

Part 2. We adapt the recursive technique devised in Friesz et al. (1993). Let $X^{(n)}(\cdot), n \geq 1$, and $X(\cdot)$ denote the arc volumes corresponding to $U^{(n)}(\cdot), n \geq 1$ and $U(\cdot)$, respectively. Assume, without loss of generality, that

$$
X^{(n)}\left(t_{0}\right)=0, \quad X\left(t_{0}\right)=0
$$

and that, for the flow profile $U(\cdot)$, the first vehicle enters the arc of interest at time $t_{0}$. In addition, let $t_{1}$ denote the time that first vehicle exits the arc of interest. By definition

$$
t_{1}=D(0)=\beta
$$


For all $t \in\left[t_{0}, t_{1}\right]$, since no vehicle can exit the arc before time $t_{1}$, we have

$$
X^{(n)}(t)=U^{(n)}(t) \quad X(t)=U(t) \quad t \in\left[t_{0}, t_{1}\right]
$$

For each flow profile $U^{(n)}, n \geq 1$, denote the exit time function restricted to $\left[t_{0}, t_{1}\right]$ by $\tau_{1}^{(n)}(\cdot)$. Under the flow profile $U$, denote the exit time function restricted to $\left[t_{0}, t_{1}\right]$ by $\tau_{1}(\cdot)$. Then

$$
\begin{gathered}
\tau_{1}^{(n)}(t)=t+D\left(X^{(n)}(t)\right)=t+a U^{(n)}(t)+\beta, \quad t \in\left[t_{0}, t_{1}\right] \\
\tau_{1}(t)=t+D(X(t))=t+a U(t)+\beta, \quad t \in\left[t_{0}, t_{1}\right]
\end{gathered}
$$

We conclude that $\tau_{1}^{(n)} \longrightarrow \tau_{1}$ uniformly on $\left[t_{0}, t_{1}\right]$. Now let

$$
\tilde{t}_{2} \doteq \inf _{n} \tau_{1}^{(n)}\left(t_{1}\right) \leq \tau_{1}\left(t_{1}\right)
$$

Fix $\delta$ small enough and call $t_{2} \doteq \tilde{t}_{2}-\delta$. See Fig. 1 for a graphical illustration of these notations. By Theorem $\mathrm{i}$, the functions $\left(\tau_{1}^{(n)}\right)^{-1}, n \geq 1$, and $\tau_{1}^{-1}$, are well-defined, continuous and strictly increasing. We claim that $\left\{\left(\tau_{1}^{(n)}\right)^{-1}\right\}_{n \geq 1}$ uniformly converge to $\tau_{1}^{-1}$ on $\left[t_{1}, t_{2}\right]$. To see this, we need to extend the arrival time function $\tau_{1}$ and $\tau_{1}^{(n)}$ to the interval $\left(-\infty, t_{0}\right)$. Because no vehicle is present during $\left(-\infty, t_{0}\right)$, it is natural to assign

$$
\tau_{1}(t)=t+\beta, \quad \tau^{(n)}(t)=t+\beta
$$

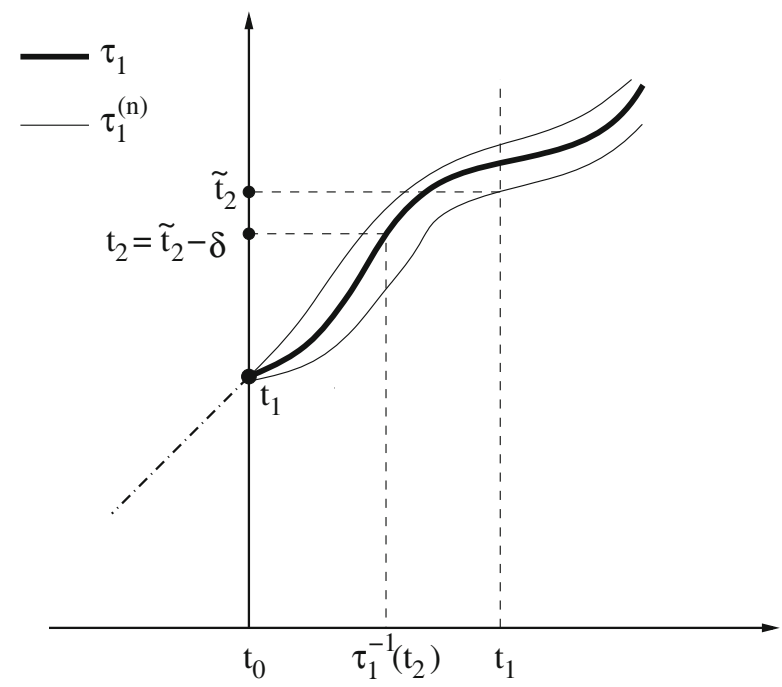

Fig. 1 Definitions of $\tilde{t}_{2}$ and $t_{2}$ 
This means, if an infinitesimal flow particle enters the arc at $t \in\left(-\infty, t_{0}\right)$, its travel delay will always be $\beta$. Fix any $\varepsilon<t_{1}-\tau_{1}^{-1}\left(t_{2}\right)$, and consider the following quantities:

$$
\Delta_{\varepsilon}^{-} \doteq \inf _{t \in\left[t_{0}, \tau_{1}^{-1}\left(t_{2}\right)\right]}\left\{\tau_{1}(t)-\tau_{1}(t-\varepsilon)\right\} \quad \Delta_{\varepsilon}^{+} \doteq \inf _{t \in\left[t_{0}, \tau_{1}^{-1}\left(t_{2}\right)\right]}\left\{\tau_{1}(t+\varepsilon)-\tau_{1}(t)\right\}
$$

Since the infimum of a continuous function on a compact interval must be obtained at some point $t \in\left[t_{0}, \tau_{1}^{-1}\left(t_{2}\right)\right]$, we conclude $\Delta_{\varepsilon}^{-}, \Delta_{\varepsilon}^{+}>0$ by the strict monotonicity of $\tau_{1}$ established in Theorem $\mathrm{i}$.

According to the uniform convergence $\tau_{1}^{(n)} \longrightarrow \tau_{1}$ on $\left(-\infty, t_{1}\right]$, there exists some $N>0$ such that for any $n \geq N$, we have

$$
\tau_{1}^{(n)}(t) \leq \tau_{1}(t)+\Delta_{\varepsilon}^{-} / 2 \quad \tau_{1}^{(n)}(t) \geq \tau_{1}(t)-\Delta_{\varepsilon}^{+} / 2 \quad \forall t \in\left[-\infty, t_{1}\right]
$$

For any $s \in\left[t_{1}, t_{2}\right]$, we have $\tau_{1}^{-1}(s) \in\left[t_{0}, \tau_{1}^{-1}\left(t_{2}\right)\right]$. Therefor, for all $n \geq N$, in view of (28) and (27), we have

$$
\begin{aligned}
& \tau_{1}^{(n)}\left(\tau_{1}^{-1}(s)-\varepsilon\right) \leq \tau_{1}\left(\tau_{1}^{-1}(s)-\varepsilon\right)+\Delta_{\varepsilon}^{-} / 2 \leq \tau_{1}\left(\tau_{1}^{-1}(s)\right)-\Delta_{\varepsilon}^{-} / 2=s-\Delta_{\varepsilon}^{-} / 2 \\
& \tau_{1}^{(n)}\left(\tau_{1}^{-1}(s)+\varepsilon\right) \geq \tau_{1}\left(\tau_{1}^{-1}(s)+\varepsilon\right)-\Delta_{\varepsilon}^{+} / 2 \geq \tau_{1}\left(\tau_{1}^{-1}(s)\right)+\Delta_{\varepsilon}^{+} / 2=s+\Delta_{\varepsilon}^{+} / 2
\end{aligned}
$$

By the Intermediate Value Theorem, there exists some $t^{*} \in\left[\tau_{1}^{-1}(s)-\varepsilon, \tau^{-1}(s)+\right.$ $\varepsilon$ ] with $\tau_{1}^{(n)}\left(t^{*}\right)=s$. In other words, we know

$$
\left|\left(\tau_{1}^{(n)}\right)^{-1}(s)-\tau_{1}^{-1}(s)\right|=\left|t^{*}-\tau_{1}^{-1}(s)\right|<\varepsilon, \quad \forall n \geq N
$$

This finishes our claim that $\left(\tau_{1}^{(n)}\right)^{-1} \longrightarrow \tau_{1}^{-1}$ uniformly on $\left[t_{1}, t_{2}\right]$.

Let $\tau_{2}^{(n)}(\cdot), n \geq 1$ and $\tau_{2}(\cdot)$ be the exit time functions for cars entering during the interval $\left[t_{1}, t_{2}\right]$, corresponding to the entering flow profiles $U^{(n)}(\cdot), n \geq 1$ and $U(\cdot)$, respectively. Then, for each $t \in\left[t_{1}, t_{2}\right]$, we may state

$$
\begin{aligned}
\tau_{2}^{(n)}(t) & =t+a\left[U^{(n)}(t)-U^{(n)}\left(\left(\tau_{1}^{(n)}\right)^{-1}(t)\right)\right]+\beta, \quad n \geq 1 \\
\tau_{2}(t) & =t+a\left[U(t)-U\left(\tau_{1}^{-1}(t)\right)\right]+\beta
\end{aligned}
$$

Now we make the claim that $U^{(n)}\left(\left(\tau_{1}^{(n)}\right)^{-1}(t)\right) \longrightarrow U\left(\tau_{1}^{-1}(t)\right)$ uniformly on $\left[t_{1}, t_{2}\right]$. Indeed, for any $\varepsilon>0$, there exists $N_{1}>0$ such that, if $n>N_{1}$, we have

$$
\left|U^{(n)}(t)-U(t)\right|<\varepsilon / 2, \quad \forall t \in\left[t_{0}, t_{f}\right]
$$

Moreover, the functions $\left(\tau_{1}^{(n)}\right)^{-1}, n \geq 1$, and $\tau_{1}^{-1}$ have a uniformly bounded range on $\left[t_{1}, t_{2}\right]$, namely $\left[t_{0}, t_{1}\right]$. By the Heine-Cantor theorem, $U(\cdot)$ restricted to $\left[t_{0}, t_{1}\right]$ is uniformly continuous, which means we can find $\delta_{0}>0$ such that, for any $s_{1}, s_{2} \in\left[t_{0}, t_{1}\right]$ with $\left|s_{1}-s_{2}\right|<\delta_{0}$, the following holds:

$$
\left|U\left(s_{1}\right)-U\left(s_{2}\right)\right|<\varepsilon / 2
$$


By uniform convergence of $\left(\tau_{1}^{(n)}\right)^{-1} \longrightarrow \tau_{1}^{-1}$, we may choose $N_{2}>0$ so that, for $n>N_{2}$, we have

$$
\left|\left(\tau_{1}^{(n)}\right)^{-1}(t)-\tau_{1}^{-1}(t)\right|<\delta_{0}
$$

Thus we deduce that, given $n>\max \left\{N_{1}, N_{2}\right\}$, for any $t \in\left[t_{1}, t_{2}\right]$, the following is true:

$$
\begin{aligned}
& \left|U^{(n)}\left(\left(\tau_{1}^{(n)}\right)^{-1}(t)\right)-U\left(\tau_{1}^{-1}(t)\right)\right| \\
\leq & \left|U^{(n)}\left(\left(\tau_{1}^{(n)}\right)^{-1}(t)\right)-U\left(\left(\tau_{1}^{(n)}\right)^{-1}(t)\right)\right|+\left|U\left(\left(\tau_{1}^{(n)}\right)^{-1}(t)\right)-U\left(\tau_{1}^{-1}(t)\right)\right| \\
< & \varepsilon / 2+\varepsilon / 2=\varepsilon
\end{aligned}
$$

This shows the uniform convergence $U^{(n)}\left(\left(\tau_{1}^{(n)}\right)^{-1}(t)\right) \longrightarrow U\left(\tau_{1}^{-1}(t)\right)$ on $\left[t_{1}, t_{2}\right]$, and our claim is substantiated. As an immediate consequence of (29) and (30), $\tau_{2}^{(n)}$ converges to $\tau_{2}$ uniformly on $\left[t_{1}, t_{2}\right]$.

Part 3. We now proceed by induction as follows. Choose any $v \geq 2$, and call

$$
\tilde{t}_{v+1} \doteq \inf _{n} \tau_{v}^{(n)}\left(t_{v}\right), \quad t_{v+1} \doteq \tilde{t}_{v+1}-\delta
$$

where the constant $\delta$ is the same as that in Part 2. Using the induction hypothesis that $\tau_{v}^{(n)}$ converges to $\tau_{v}$ uniformly on $\left[t_{v-1}, t_{v}\right]$, we show the following uniform convergence on $\left[t_{v}, t_{v+1}\right]$ :

$$
\left(\tau_{v}^{(n)}\right)^{-1} \longrightarrow \tau_{v}^{-1}
$$

The proof is similar to what has been done in Part 2. Now, introduce $\tau_{v+1}^{(n)}(\cdot), n \geq$ 1 , and $\tau_{v+1}(\cdot)$, which are the exit time functions corresponding to $U^{(n)}(\cdot), n \geq 1$ and $U(\cdot)$ respectively; and they are restricted to the time interval $\left[t_{v}, t_{v+1}\right]$. Similar to results (29) and (30), we have that, for $t \in\left[t_{v}, t_{v+1}\right]$, the following holds:

$$
\begin{aligned}
& \tau_{\nu+1}^{(n)}(t)=t+a\left[U^{(n)}(t)-U^{(n)}\left(\left(\tau_{v}^{(n)}\right)^{-1}(t)\right)\right]+\beta \quad n \geq 1 \\
& \tau_{\nu+1}(t)=t+a\left[U(t)-U\left(\tau_{v}^{-1}(t)\right)\right]+\beta
\end{aligned}
$$

It can be shown as before that $U^{(n)}\left(\left(\tau_{v}^{(n)}\right)^{-1}\right) \rightarrow U\left(\tau_{v}^{-1}\right)$ uniformly on $\left[t_{v}, t_{v+1}\right]$. Therefore $\tau_{v+1}^{(n)} \longrightarrow \tau_{v+1}$ uniformly on $\left[t_{v}, t_{v+1}\right]$. This finishes the induction.

Part 4. We now have obtained a sequence $\left\{\left[t_{\nu}, t_{v+1}\right]\right\}_{\nu \geq 0}$ of intervals. On each interval $\left[t_{v}, t_{v+1}\right]$, the uniform convergence

$$
\tau_{v+1}^{(n)} \longrightarrow \tau_{v+1}
$$

holds. Notice that, by construction, $t_{v+1}-t_{v} \geq \beta-\delta>0, \forall v \geq 1$; therefore the interval $\left[t_{0}, t_{f}\right]$ can be covered by finitely many such intervals. As a consequence, 
we easily obtain the uniform convergence of $\tau^{(n)}(\cdot) \longrightarrow \tau(\cdot)$ on the entire horizon $\left[t_{0}, t_{f}\right]$, where $\tau^{(n)}(\cdot)$ corresponds to $U^{(n)}(\cdot), n \geq 1$ and $\tau(\cdot)$ corresponds to $U(\cdot)$.

Let $v^{(n)}(\cdot), n \geq 1$, and $v(\cdot)$ be the exit flows of the single arc and then define the cumulative exit vehicle count

$$
V(t) \doteq \int_{t_{0}}^{t} v(s) d s, \quad V^{(n)}(t) \doteq \int_{t_{0}}^{t} v^{(n)}(s) d s,
$$

It then follows immediately from the relationships

$$
V(t)=U\left(\tau^{-1}(t)\right) \quad V^{(n)}(t)=U^{(n)}\left(\left(\tau^{(n)}\right)^{-1}(t)\right)
$$

that $V^{(n)}$ converges uniformly to $V(\cdot)$ on $\left[t_{0}, t_{f}\right]$.

Part 5. Consider a general network $(\mathcal{A}, \mathcal{V})$ with a converging sequence $h^{(n)} \longrightarrow h$ in $\Lambda \subset\left(L^{2}\left(\left[t_{0}, t_{f}\right]\right)\right)^{|\mathcal{P}|}$. Define for $p \in \mathcal{P}$ the following:

$$
H_{p}^{(n)}(t) \equiv \int_{t_{0}}^{t} h_{p}^{(n)}(s), d s, \quad H_{p}(t) \doteq \int_{t_{0}}^{t} h_{p}(s) d s
$$

Then $H_{p}^{(n)}(\cdot)$ converge uniformly to $H_{p}(\cdot)$. For each arc $a \in \mathcal{A}$, where the notation employed has an obvious meaning, the cumulative entering vehicle count $U_{a}^{(n)}(\cdot)$ is given by

$$
U_{a}^{(n)}(t)=\sum_{p} H_{p}^{(n)}(t)+\sum_{a^{\prime} \in \mathcal{I}(a)} V_{a^{\prime}}^{(n)}(t)
$$

In the above, the first summation is over all paths that use $a$ as the first arc; in the second summation, $\mathcal{I}(a)$ denotes the set of arcs immediately upstream from arc a. A simple mathematical induction with the results established in previous steps yields the uniform convergence

$$
U_{a}^{(n)}(t) \longrightarrow U_{a}(t), \quad V_{a}^{(n)}(t) \longrightarrow V_{a}(t), \quad \tau_{a}^{(n)}(t) \longrightarrow \tau_{a}(t), \quad \forall a \in \mathcal{A}
$$

where $\tau_{a}(\cdot)$ is the exit time function of $\operatorname{arc} a$. Thus, for each path $p \in \mathcal{P}$, the path delay $D_{p}\left(\cdot, h^{(n)}\right)$ also converges uniformly to $D_{p}(\cdot, h)$ since it is a finite sum of arc delays. It remains to show that the effective delays obey $\Psi_{p}\left(\cdot, h^{(n)}\right) \longrightarrow$ $\Psi_{p}\left(\cdot, h^{(n)}\right)$ uniformly on $\left[t_{0}, t_{f}\right]$. Recall

$$
\Psi_{p}(t, h)=D_{p}(t, h)+\mathcal{F}\left(t+D_{p}(t, h)-T_{A}\right)
$$

Notice that $\mathcal{F}$ is uniformly continuous on $\left[t_{0}, t_{f}\right]$ by the Heine-Cantor theorem; this means, for any $\varepsilon>0$, there exists $\sigma>0$ such that whenever $\left|s_{1}-s_{2}\right|<\sigma$, we have

$$
\left|\mathcal{F}\left(s_{1}\right)-\mathcal{F}\left(s_{2}\right)\right|<\varepsilon / 2
$$

Moreover, by uniform convergence, there exits $N>0$ such that, for all $n>N$, we have

$$
\left|D_{p}\left(t, h^{(n)}\right)-D_{p}(t, h)\right|<\min \{\sigma, \varepsilon / 2\} \quad \forall t \in\left[t_{0}, t_{f}\right]
$$


We then readily deduce that, given $n>N$, the following holds for all $t \in\left[t_{0}, t_{f}\right]$ :

$$
\begin{aligned}
& \left|\Psi_{p}\left(t, h^{(n)}\right)-\Psi_{p}(t, h)\right| \\
\leq & \left|D_{p}\left(t, h^{(n)}\right)-D_{p}(t, h)\right|+\left|\mathcal{F}\left(t+D_{p}\left(t, h^{(n)}\right)-T_{A}\right)-\mathcal{F}\left(t+D_{p}(t, h)-T_{A}\right)\right| \\
< & \varepsilon / 2+\varepsilon / 2=\varepsilon
\end{aligned}
$$

Final Argument Finally, uniform convergence on the compact interval $\left[t_{0}, t_{f}\right]$ implies convergence in the $L^{2}$ norm:

$$
\int_{t_{0}}^{t_{f}}\left(\Psi_{p}\left(t, h^{(n)}\right)-\Psi_{p}(t, h)\right)^{2} d t \longrightarrow 0, \quad n \longrightarrow \infty, \quad p \in \mathcal{P}
$$

Summing up (33) over $p \in \mathcal{P}$, we obtain the convergence $\Psi\left(\cdot, h^{(n)}\right) \longrightarrow \Psi(\cdot, h)$ in the Hilbert space $\left(L^{2}\left(\left[t_{0}, t_{f}\right]\right)\right)^{|\mathcal{P}|}$.

\section{Concluding Remarks}

Dynamic traffic assignment differs from static traffic assignment in that path delay does not enjoy a closed form. In fact the path delays needed for the study of dynamic user equilibria (DUE) are operators that may only be specified numerically. Moreover, such path delay operators are based on the specific model of arc delay employed for network loading. We have considered in this paper path delay operators for the network loading procedure that is endogenous to Friesz et al. (1993), which has been referred to as the link delay model (LDM). We have shown that the LDM-based path delay operators are strongly continuous under the very mild assumption that the link traversal time function is affine. In addition, our proof of continuity relies on no ad hoc assumptions on the uniform boundedness of the path flows. Combined with the results, Browder (1968), Zhu and Marcotte (2000), Szeto (2003), and Han et al. (2013a, b, c, 2016), an increasingly clear understanding of DUE based on different network performance models is emerging.

Acknowledgements This work is partially supported by NSF through grant EFRI-1024707, A theory of complex transportation network design.

Open Access This article is distributed under the terms of the Creative Commons Attribution 4.0 International License (http://creativecommons.org/licenses/by/4.0/), which permits unrestricted use, distribution, and reproduction in any medium, provided you give appropriate credit to the original author(s) and the source, provide a link to the Creative Commons license, and indicate if changes were made.

\section{References}

Ben-Akiva M, Gao S, Lu L, Wen Y (2015) Combining disaggregate route choice estimation with aggregate calibration of a dynamic traffic assignment model. Networks and Spatial Economics 15(3):559-581

Browder FE (1968) The fixed point theory of multi-valued mappings in topological vector spaces. Math Ann 177:283-301 
Carey M (2001) Dynamic traffic assignment with more flexible modelling within links. Networks and Spatial Economics 1(3):349-375

Carey M, Ge Y (2012) Comparison of methods for path flow reassignment for dynamic user equilibrium. Networks and Spatial Economics 12(3):337-376

Friesz TL, Bernstein D, Smith T, Tobin R, Wie B (1993) A variational inequality formulation of the dynamic network user equilibrium problem. Oper Res 41(1):80-91

Friesz TL, Bernstein D, Suo Z, Tobin R (2001) Dynamic network user equilibrium with state-dependent time lags. Network and Spatial Economics 1(3):319-347

Friesz TL, Kim T, Kwon C, Rigdon MA (2010) Approximate network loading and dual-time-scale dynamic user equilibrium. Transp Res B 45:176-207

Friesz TL, Han K, Neto PA, Meimand A, Yao T (2013) Dynamic user equilibrium based on a hydrodynamic model. Transp Res B 47:102-126

Friesz TL, Meimand A (2014) Dynamic user equilibria with elastic demand. Transportmetrica A: Transport Science 10(7):661-668

Friesz TL, Mookherjee R (2006) Solving the dynamic network user equilibrium problem with statedependent time shifts. Transp Res B 40(3):207-229

Han D, Lo HK (2002) Two new self-adaptive projection methods for variational inequality problems. Comput Math Appl 43:1529-1537

Han K, Friesz TL, Yao T (2013a) A partial differential equation formulation of Vickrey's bottleneck model, part I: methodology and theoretical analysis. Transp Res B 49:55-74

Han K, Friesz TL, Yao T (2013b) A partial differential equation formulation of Vickrey's bottleneck model, part II: numerical analysis and computation. Transp Res B 49:75-93

Han K, Friesz TL, Yao T (2013c) Existence of simultaneous route and departure choice dynamic user equilibrium. Transp Res B 53:17-30

Han K, Friesz TL, Szeto WY, Liu H (2015a) Elastic demand dynamic network user equilibrium: formulation, existence and computation. Transp Res B 81:183-209

Han K, Piccoli B, Friesz TL (2016) Continuity of the path delay operator for dynamic network loading with spillback. Transp Res B 92:211-233

Han K, Szeto WY, Friesz TL (2015b) Formulation, existence, and computation of boundedly rational dynamic user equilibrium with fixed or endogenous user tolerance. Transp Res B 79:16-49

Han D, Lo HK (2003) Solving nonadditive traffic assignment problems: a decent method for co-coercive variational inequalities. Eur J Oper Res 159:529-544

Kachroo P, Özbay K (2005) Feedback control solutions to network level user-equilibrium real-time dynamic traffic assignment problems. Networks and Spatial Economics 5(3):243-260

Konnov IV (2003) Application of the proximal point method to non monotone equilibrium problems. J Optim Theory Appl 119:317-333

Lo HK, Szeto WY (2002) A cell-based dynamic traffic assignment model: formulation and properties. Math Comput Model 35(7-8):849-865

Goya-Gómez B, Salas-Olmedo MH, García-Palomares JC, Gutiérrez J (2017) Dynamic accessibility using big data: the role of the changing conditions of network congestion and destination attractiveness. Networks and spatial economics. https://doi.org/10.1007/s11067-017-9348-Z

Nie X, Zhang H (2005) A comparative study of some macroscopic link models used in dynamic traffic assignment. Networks and Spatial Economics 5:89-115

Nesterov Y, De Palma A (2003) Stationary dynamic solutions in congested transportation networks: summary and perspectives. Networks and Spatial Economics 3(3):371-395

Nie Y, Zhang HM (2010) Solving the dynamic user optimal assignment problem considering queue spillback. Networks and Spatial Economics 10:49-71

Pang J, Han L, Ramadurai G, Ukkusuri S (2011) A continuous-time linear complementarity system for dynamic user equilibria in single bottleneck traffic flows. Mathematical Programming Series A 133(12):437-460

Peeta S, Ziliaskopoulos A (2001) Foundations of dynamic traffic assignment: the past, the present and the future. Networks and Spatial Economics 1(3/4):233-265

Szeto WY (2003) Dynamic traffic assignment: formulations, properties, and extensions. PhD thesis, The Hong Kong University of Science and Technology, China

Szeto WY, Lo HK (2004) A cell-based simultaneous route and departure time choice model with elastic demand. Transp Res B 38:593-612 
Szeto WY, Lo HK (2006) Dynamic traffic assignment: properties and extensions. Transportmetrica 2(1):31-52

Ukkusuri S, Han L, Doan K (2012) Dynamic user equilibrium with a path based cell transmission model for general traffic networks. Transp Res B 46(10):1657-1684

Wardrop J (1952) Some theoretical aspects of road traffic research. In: ICE proceedings: part II, engineering divisions, vol 1, pp 325-362

Wie BW, Tobin RL, Carey M (2002) The existence, uniqueness and computation of an arc-based dynamic network user equilibrium formulation. Transp Res B 36(10):897-918

Zhang HM, Nie X (2005) Some consistency conditions for dynamic traffic assignment problems. Networks and Spatial Economics 5(1):71-87

Zhu DL, Marcotte P (2000) On the existence of solutions to the dynamic user equilibrium problem. Transp Sci 34(4):402-414 